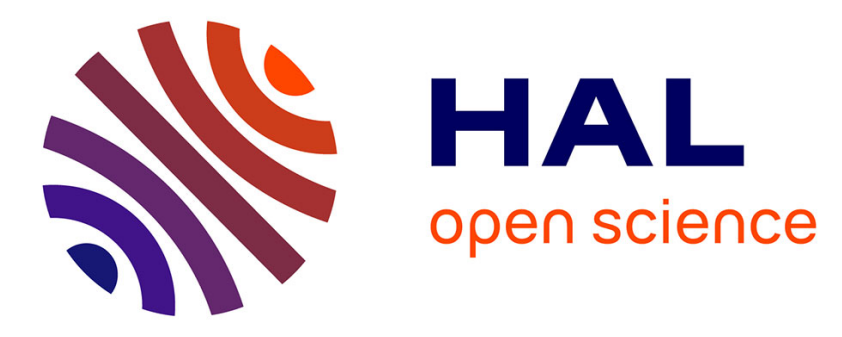

\title{
Pressure gradient effect on spin-crossover materials: Experiment vs theory
}

Ionela Rusu, Ioan Cozmin Manolache-Rusu, Andrei Diaconu, Oleg Palamarciuc, Il'ya A Gural'Skiy, Gábor Molnár, Aurelian Rotaru

\section{- To cite this version:}

Ionela Rusu, Ioan Cozmin Manolache-Rusu, Andrei Diaconu, Oleg Palamarciuc, Il'ya A Gural'Skiy, et al.. Pressure gradient effect on spin-crossover materials: Experiment vs theory. Journal of Applied Physics, 2021, 129 (6), pp.064501. 10.1063/5.0042582 . hal-03179332

\section{HAL Id: hal-03179332 \\ https://hal.science/hal-03179332}

Submitted on 24 Mar 2021

HAL is a multi-disciplinary open access archive for the deposit and dissemination of scientific research documents, whether they are published or not. The documents may come from teaching and research institutions in France or abroad, or from public or private research centers.
L'archive ouverte pluridisciplinaire HAL, est destinée au dépôt et à la diffusion de documents scientifiques de niveau recherche, publiés ou non, émanant des établissements d'enseignement et de recherche français ou étrangers, des laboratoires publics ou privés. 


\section{Pressure Gradient Effect on Spin-Crossover Materials: Experiment vs. Theory}

\section{Ionela Rusu¹, Ioan Cosmin Manolache-Rusu², Andrei Diaconu', Oleg Palamarciuc ${ }^{3,4}$, Il'ya A.}

Gural'skiy $^{5,6}$, Gabor Molnar ${ }^{7}$, Aurelian Rotaru, ${ }^{1, *}$

${ }^{1}$ Faculty of Electrical Engineering and Computer Science \& Research Center MANSiD, Stefan cel Mare University, Suceava 720229, Romania

${ }^{2}$ Faculty of Mechanical Engineering, Mechatronics and Management \& Research Center MANSiD, Stefan cel Mare University, Suceava 720229, Romania

${ }^{3}$ Polivalent-95 SRL, Chisinau, MD-2028, Moldova

${ }^{4}$ Moldova State University, Institute of Research and Innovation, A. Mateevici Street 60, MD-2009 Chisinau, Republic of Moldova

${ }^{5}$ Department of Chemistry, Taras Shevchenko National University of Kyiv, 64/13 Volodymyrska St., 01601 Kyiv, Ukraine

${ }^{6}$ UkrOrgSyntez Ltd., 67 Chervonotkatska St., 02094 Kyiv, Ukraine.

${ }^{7}$ Laboratoire de Chimie de Coordination, CNRS UPR-8241 and Université de Toulouse, UPS, INP, F-31077 Toulouse, France.

* Corresponding author: aurelian.rotaru@usm.ro

\section{Abstract}

We studied the effect of a non-hydrostatic pressure on the hysteretic spin crossover in coordination complexes. By introducing into an Ising-like model a double distribution of the interactions and gap energy, respectively, we were able to generate the major hysteresis loop and the FORC diagram for spin-crossover systems of $10^{6}$ hysterons (like-spin domains). We show that, for high pressure gradients around the spin-crossover system, the thermal hysteresis loop takes an asymmetric shape, in good agreement with the experimental data on pressure effect recorded at low temperatures, below the solidification of the pressure transmitting medium. Interestingly, the FORC diagram method seems to be much more sensitive to local changes than the "bulk" parameters, which characterize the major hysteresis loop.

\section{Introduction}

Spin-crossover (SCO) complexes form an interesting class of switchable materials that can exist in two different spin states, known as the low-spin (LS) and high-spin (HS) states. These complexes may be switched between 
the two spin states under various stimuli including temperature, pressure, light, magnetic and electrical fields [1, 2]. Among these stimuli, pressure is one of the most appealing thermodynamic parameters used to probe the spin transition properties of spin-crossover materials since it allows for tuning both the crystal field as well as the interactions between SCO centers. Since the pioneering works of Drickamer [3, 4], König [5] and Gütlich,[6, 7], the pressure effect has continuously attracted the interest of the scientific community, being intensively studied until nowadays. The pressure effect on spin-crossover materials has been investigated using various techniques, such as Mössbauer spectroscopy [5], X-Ray and neutron diffraction [8-13], X-Ray spectroscopies [14, 15], Raman spectroscopy [16, 17], optical reflectivity [18-25], magnetometry [26-31] and dielectric spectroscopy [32]. In general, the application of an external pressure induces a transformation of the SCO complex to the low-spin state, i.e. to the state of lower molecular volume, and has the effect of shifting the switching temperatures towards higher values. However, the interpretation of the pressure effect on molecular SCO phenomena has been, in some cases, compromised by the relatively poor or incomplete information resulted from experimental difficulties related to the need for hydrostatic conditions at low temperatures. The practical implementation consists of immersing the sample in a pressure transmitting fluid meant to support no shear. However, the melting point of fluids is usually increased under pressure and solidification inevitably occurs at some pressure. Beyond this point, the pressure across the experimental volume is generally inhomogeneous and differential as well as shear stresses appear [33]. Depending on the type of measurement, this can lead to a more or less dramatic decrease in the quality and accuracy of the data and often to the presence of 'anomalies', which might be erroneously ascribed to new physical phenomena. For example, the hysteretic properties of spin-crossover microparticles, embedded in glass-forming or semicrystalline matrices, reveal a larger hysteresis loop as compared to the bulk $[34,35]$. This effect has been attributed to an increase of the cooperativity of the SCO system due to particle-matrix interactions. An indispensable condition for generating a high-quality hydrostatic pressure is that the pressure transmitting medium should be a fluid in the whole experimental P-T range. When the pressure homogeneity is poor, it is sometimes difficult to distinguish real coexistence of two phases from a simple mixture of two phases due to pressure distribution around the sample. This adversity is likely to occur when the pressure region under discussion is beyond the solidification of the pressure medium.

The purpose of the present study is to investigate the effect of a non-hydrostatic pressure on the thermally induced spin transition in spin-crossover complexes.

\section{Theory: the Ising-like model}

A useful microscopic model of SCO, the so-called Ising-like model, has been proposed by Bousseksou et al. [36] nearly 3 decades ago. This approach takes into account the degenerate energy levels and it was successfully used 
to describe both static and dynamic properties of SCO compounds under different external stimuli (temperature, pressure, magnetic and electric fields) [37, 38]. The Hamiltonian in the Ising-like model can be written as follows:

$$
H=\frac{1}{2} \sum_{(i)}\left(\Delta-k_{B} T \ln g\right) \hat{\sigma}_{i}-\sum_{<i, j>} J_{i j} \hat{\sigma}_{i} \hat{\sigma}_{j}-G\langle\hat{\sigma}\rangle \sum_{(i)} \hat{\sigma}_{i}
$$

where $\hat{\sigma}_{i}$ is the fictitious spin operator associated to molecule $i$ having the eigenvalues +1 (when the molecule is in the HS state) and -1 (when the molecule is in the LS state), $\Delta$ is the energy gap between the HS and LS states, $k_{B}$ is Boltzmann constant $\left(=1.38064852(79) \times 10^{-23} \mathrm{~J} \cdot \mathrm{K}^{-1}\right), g$ is the degeneracy ratio between HS and LS energy levels (>1), $J_{i j}$ stands for the short-range interaction parameter representing the cooperative interaction that only exists between the nearest neighbouring pairs ( $J_{i j}$ includes the nearest neighbors number), $<i, j>$ denotes the fact that the sum is only made over the nearest neighbors pairs, $G$ stands for a long range interaction parameter that is expressed here in a mean-field approach with $\langle\hat{\sigma}\rangle$ being the average fictitious spin.

The pressure effect has been introduced considering that the energy gap is changing due to the elastic energy change $p \cdot \delta V$ when increasing the applied pressure [3]:

$$
\Delta(p)=\Delta(p=0)+p \delta V
$$

where $\delta V$ is the volume variation of the SCO molecules during the spin transition phenomenon and $p$ is the externally applied pressure. In the mean-field approach, the Hamiltonian $\mathrm{H}_{\mathrm{MF}}$ can be written as follows:

$$
H_{M F}=\frac{\Delta(p)-k_{B} T \ln g}{2} \hat{\sigma}-(q J+G) \hat{\sigma}<\hat{\sigma}>;
$$

where $q$ is the number of nearest neighbours. The average fictitious spin $\langle\hat{\sigma}\rangle$ of a hysteron will be further denoted by $m$ that can be expressed as:

$$
m=\tanh \left(\frac{(q J+L) m}{k_{B} T}+\frac{k_{B} T \ln g-\Delta(p)}{2 k_{B} T}\right)
$$

For the sake of simplicity, in the following calculations we use several normalized parameters: the energy gap $\Delta=\Delta(p) / k_{B}$, the interaction parameter $\Gamma=(q J+L) / k_{B}$ (which includes both short and long range interactions), the volume variation, $\alpha=\delta V \cdot 10^{-5} / k_{B}$ (where the factor $10^{-5}$ stands for the conversion of the pressure units from Pascal to bar), and the high spin fraction $n_{H S}=(m+1) / 2$. 
In order to closer represent a real system, we considered a spin crossover system of $10^{6}$ hysterons (i.e. spin-like domains), characterized by a double Gaussian distribution of energy gaps and interaction parameters of the form:

$$
P(J, \Delta)=\frac{1}{2 \pi \sigma_{J} \sigma_{\Delta}} \exp \left(\frac{-\left(J-J_{0}\right)^{2}}{2 \sigma_{J} \sigma_{\Delta}}\right) \exp \left(\frac{-\left(\Delta-\Delta_{0}\right)^{2}}{2 \sigma_{J} \sigma_{\Delta}}\right)
$$

Thus, the high spin fraction can simply be calculated by using the normalization condition:

$$
n_{H S}=\frac{\sum_{i}^{N} P(J, \Delta) \cdot n_{H S}(J, \Delta)}{N}
$$

Due to the high number of hysterons for our system, we employed high performance calculation (HPC) by using a Nvidia GeForce GTX760 GPU (GPU = Graphics Processing Unit) with a parallel architecture. Thus, the 1152 hysterons have been simultaneously simulated by using the 1152 CUDA cores of the GPU, allowing us to reasonably reduce the calculation time (from $14 \mathrm{~h}$ using sequential compilation to $20 \mathrm{msec}$. using HPC) of such systems with a macroscopic number of hysterons, close to that of a real system.

\section{Results and discussions}

The pressure gradient has been naturally introduced into the Ising-like model through the standard deviation of the reduced energy gap distribution, $\sigma(\Delta)$, expressed in Kelvin. Figure 1 shows the temperature dependence of the high spin fraction, nHs, simulated for various values of the pressure gradient and different values of the effective interaction parameter.
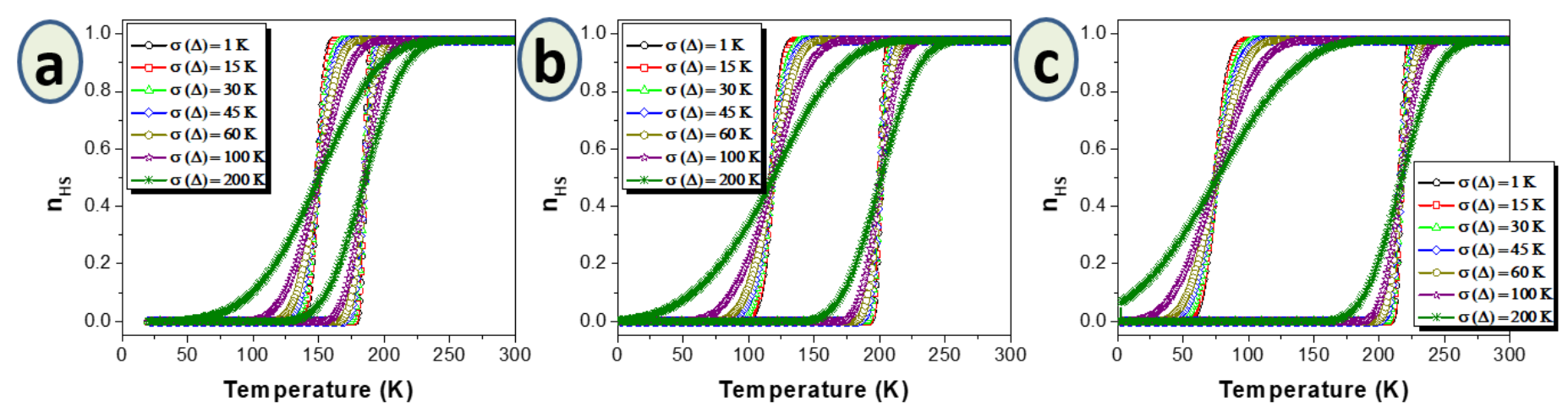

Figure 1. Thermal hysteresis simulated for various values of the pressure gradient and different values of the effective interaction parameter: (a) $\Gamma_{0}=300 \mathrm{~K}$; (b) $\Gamma_{0}=400 \mathrm{~K}$ and (c) $\Gamma_{0}=500 \mathrm{~K}$ respectively. The following computational parameters have been used: $\Delta_{0}=1200 \mathrm{~K}, \ln g=7$. 
We can see that for low pressure gradients, i.e. under hydrostatic pressure, the thermal hysteresis loop has a symmetric shape and becomes strongly asymmetric as the pressure gradient is increased. The same effect, i.e. asymmetric thermal hysteresis loop for a high pressure gradient, has been observed for various values of the interaction parameters. Such effects have been experimentally observed mostly in magnetic measurements under pressure performed on complexes characterized by low switching temperatures [6] or in nanoparticles embedded in various matrixes [34, 35]. Indeed, in magnetic measurements, a clamp-type pressure cell is typically used [39]. In such pressure cells, the pressure is transmitted to the sample through silicon oil such as Daphne 7373 or Flourinert 77/70.

\subsection{Experiment vs Theory}

In most of the previous works published on this topic, the hydrostatic character of the pressure cell is evaluated from the abruptness of the magnetic transition curve (from diamagnetic to superconductive state) of a superconducting manometer (usually $\mathrm{Pb}$ or $\mathrm{Sn}$ thin wires). In this context, we measured the superconducting transition of a $\mathrm{Pb}$ wire by using two pressure media: distilled water and Daphne 7373 (see Figure S1 in ESI). Surprisingly, the transition curves recorded on $\mathrm{Pb}$ in both water and Daphne 7373 have the same shape. However, a shift to lower values of the transition temperature of $\mathrm{Pb}$ in water, compared with that one of $\mathrm{Pb}$ in Daphne 7373 has been observed. This denotes that water applies a higher pressure around the $\mathrm{Pb}$ wire due to its negative coefficient of compressibility.

We have investigated the pressure effect on the magnetic properties of $\left[\mathrm{Fe}(\mathrm{PM}-\mathrm{BiA})_{2}(\mathrm{NCS})_{2}\right]$ spin-crossover complex by using a clamp pressure cell. The choice of this complex has been motivated by the low values of the switching temperatures (around $170 \mathrm{~K}$ ), bellow the melting point of the pressure transmitting medium. The sample was sealed in a Teflon sample holder surrounded by Daphne 7373 as the pressure transmitting medium. The magnetic measurements were performed using a MPMS3 SQUID magnetometer (Quantum Design Inc., USA), in DC mode, under a static magnetic field of 1000 Oe with a temperature sweep rate of $2 \mathrm{~K} / \mathrm{min}$. The pressure value inside the pressure cell has been determined by measuring the superconducting transition temperature of a $\mathrm{Pb}$ wire. 

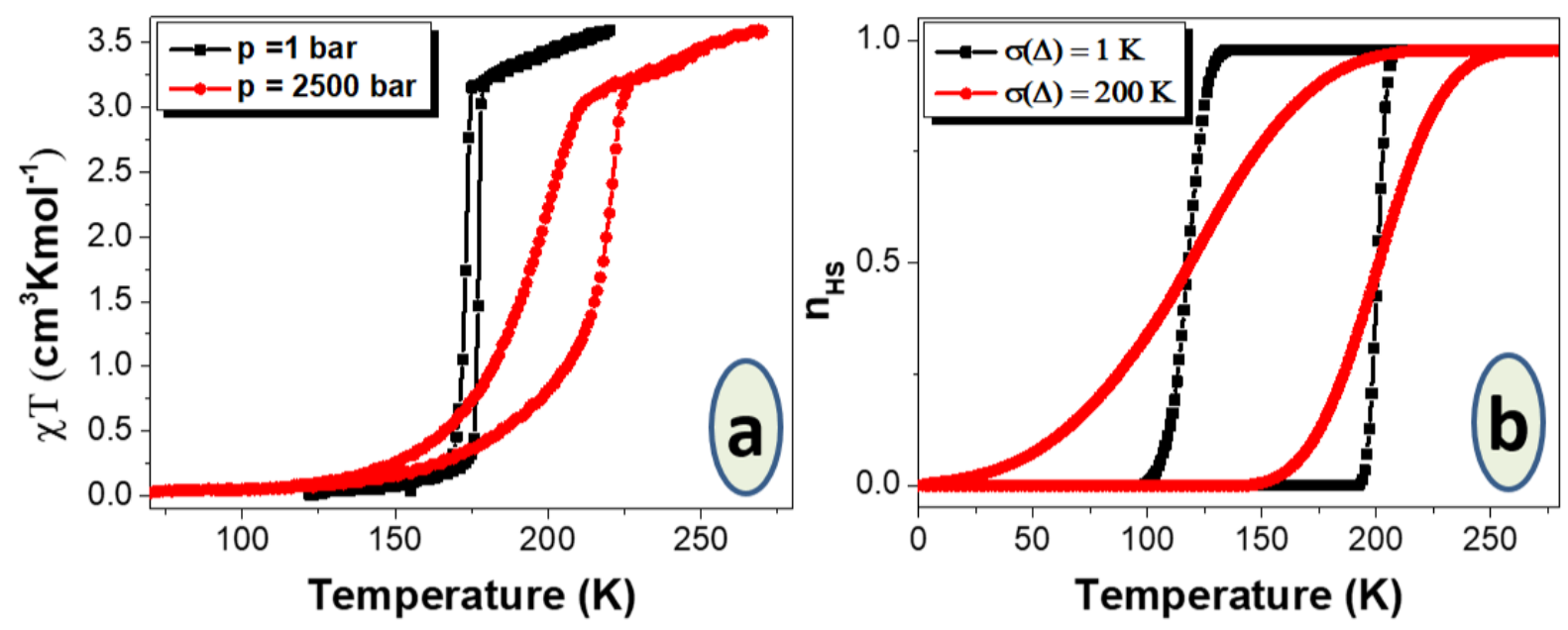

Figure 2. (a) Temperature dependence of the magnetic susceptibility recorded on [Fe( $\left.\mathrm{PM}-\mathrm{BiA})_{2}(\mathrm{NCS})_{2}\right]$ polymorph I, at various pressures. (b) Simulated thermal hysteresis curves for different pressure gradients.

At ambient pressure, $\left[\mathrm{Fe}(\mathrm{PM}-\mathrm{BiA})_{2}(\mathrm{NCS})_{2}\right]$ spin crossover complex (polymorph I) exhibits a complete LS $(\mathrm{S}=0) \leftrightarrow H S(S=2)$ thermal spin transition. By increasing the pressure, the $\left[\mathrm{Fe}(\mathrm{PM}-\mathrm{BiA})_{2}(\mathrm{NCS})_{2}\right]$ spincrossover complex (polymorph I) shows a wider, more asymmetric thermal hysteresis loop (Fig. 2a). A similar behavior was noticed in our simulations for higher values of the pressure gradient (Fig. 2b). Thus, the asymmetric shape of the thermal hysteresis loop recorded on the $\left[\mathrm{Fe}(\mathrm{PM}-\mathrm{BiA})_{2}(\mathrm{NCS})_{2}\right]$ complex might be attributed to the presence of pressure gradient around the sample. Indeed, Daphne 7373 has a freezing/melting point, at ambient pressure, of ca. $180 \mathrm{~K}$ (see Figure S2 in the ESI) which increases with increasing applied pressure. It is worth noting herein that the $\left[\mathrm{Fe}(\mathrm{PM}-\mathrm{BiA})_{2}(\mathrm{NCS})_{2}\right]$ spin crossover complex (polymorph I) under external pressure undergoes a crystallographic phase transition [6,21], which can amplify the deformation of the hysteresis loop. For a more in depth analysis of this effect, we employed the first-order reversal curve (FORC) diagram method.

\subsection{FORC diagram method}

The FORC diagram method has been proposed as an alternative characterization tool to the classical major hysteresis loop (MHL) analysis [40]. It is already known that the FORC diagram method provides detailed information from within the major hysteresis loop and enables determination of the distribution of switching temperatures and interaction fields for all "particles" (such as the like-spin mono-domains) that contribute to the hysteresis loop [23, 41-44]. In the case of a temperature induced spin transition, the FORCs are a specific class of minor hysteresis loops, for which the sweep of the temperature is reversed at a specific temperature, $\mathrm{T}_{\mathrm{a}}$, on one of the branches of the major thermal hysteresis loop, back to a specific temperature at which the domain structure is saturated. The temperature steps are chosen such that $T_{a}$ and $T_{b}$ are regularly spaced, which means that 
$n_{H S}\left(T_{a}, T_{b}\right)$ can be plotted on a regular grid. The FORC distribution $\rho\left(T_{a}, T_{b}\right)$ is defined as the mixed second derivative $\rho\left(T_{a}, T_{b}\right)=-\frac{\partial^{2} n_{H S}\left(T_{a}, T_{b}\right)}{\partial T_{a} \partial T_{b}}$.

The FORC distribution is determined at each point by fitting $n_{H S}\left(T_{a}, T_{b}\right)$ with a mixed second-order polynomial of the form $a_{1}+a_{2} T_{a}+a_{3} T_{b}+a_{4} T_{a}^{2}+a_{5} T_{b}^{2}+a_{6} T_{a} T_{b}$ to a local moving grid. In this case the value of $-\mathrm{a}_{6}$ provides the mixed second derivative of the fitted surface and it can be assigned to the center of grid as a representation of the density of the FORC distribution $\rho\left(T_{a}, T_{b}\right)$ at that point. A FORC diagram is a contour plot of a FORC distribution with $\mathrm{T}_{\mathrm{a}}=\mathrm{T}_{\mathrm{up}}$, and $\mathrm{T}_{\mathrm{b}}=\mathrm{T}_{\text {down }}$ on the vertical and horizontal axes, respectively.

Figure 3 shows the FORC diagram simulated for various pressure gradients. As it can be seen, the FORC diagrams are strongly influenced by the occurrence of a pressure gradient. For hydrostatic pressure, i.e. a low value of $\sigma(\Delta)=1 K$, the system is defined by a narrow FORC diagram characterized by a negative correlation parameter.
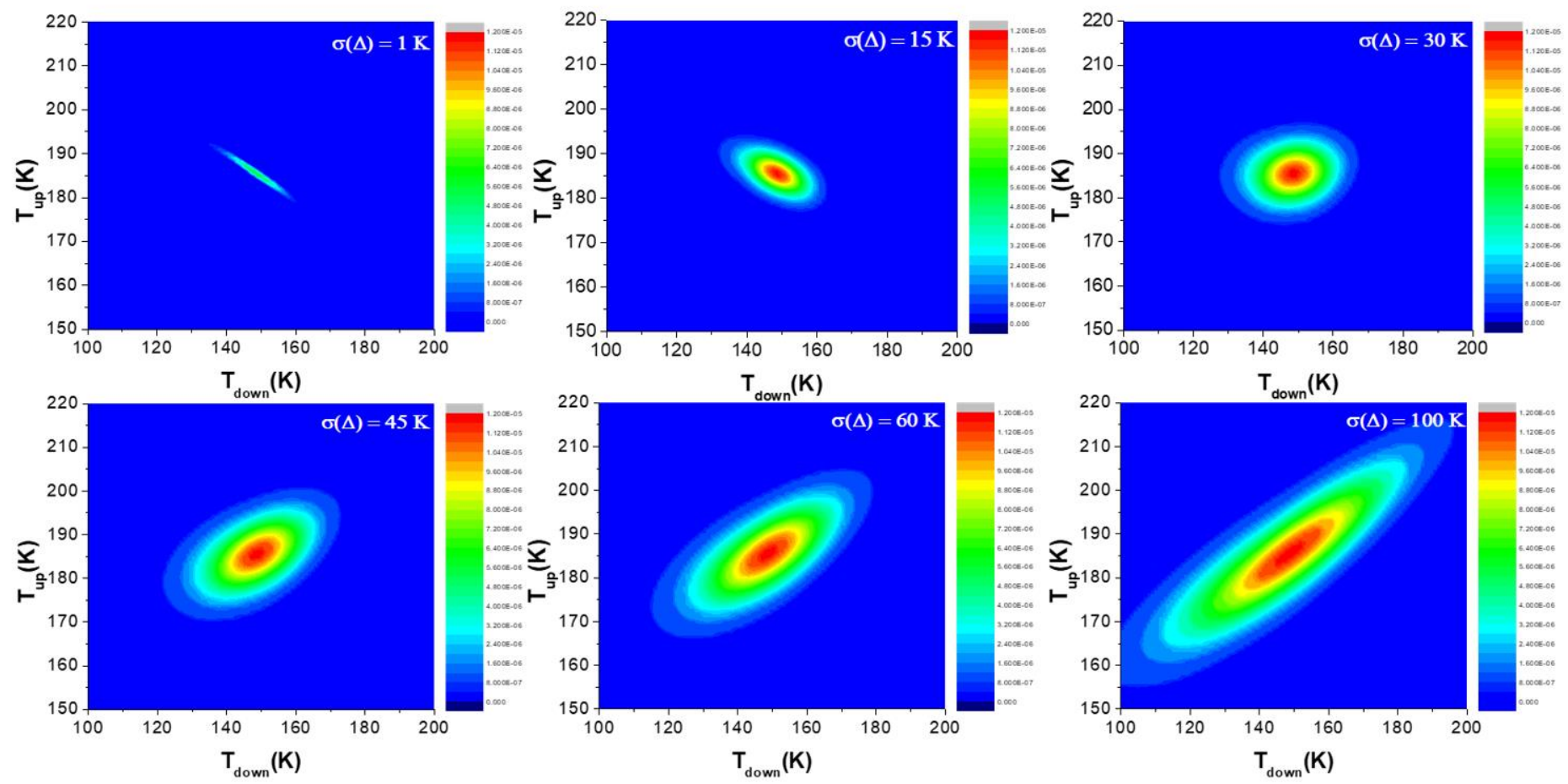

Figure 3. FORC diagrams simulated for various values of the pressure gradient. The following computational parameters have been used: $\Delta_{0}=1200 \mathrm{~K}, \Gamma_{0}=300 \mathrm{~K}$ and $\ln g=7$.

The dependence of the statistical parameters of the FORC diagrams as a function of pressure gradients is presented in Figure 4. As the pressure gradient is increased the standard deviation of the switching temperature distributions is increasing (broader FORC diagram). The correlation between the two switching temperature distributions is dramatically influenced as well. The correlation parameter of the FORC distribution is changed from -0.9 , value 
similar with that one experimentally recorded in the 1D spin crossover compounds, at ambient pressure [45, 46], to +0.9 for high pressure gradients.
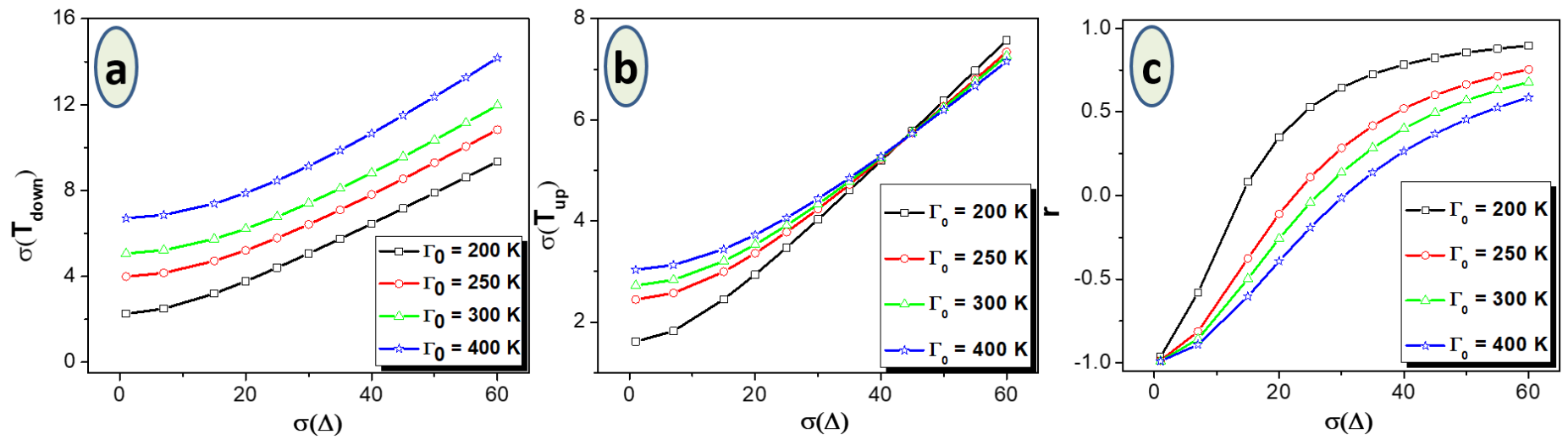

Figure 4. Pressure gradient influence on the statistical parameters obtained from the statistical analysis of the simulated FORC diagrams, for various values of the effective interaction parameter.

\subsection{MHL vs FORC}

A remarkable finding that we would like to highlight here is the sensitivity of the FORC diagram method regarding small local changes compared to the classical major hysteresis loop. Figure 5 shows the effect of small pressure gradients on the thermal hysteresis loop and on the FORC diagram, respectively. If the major hysteresis loop is only slightly affected by a pressure gradient of $\sigma(\Delta)=30 \mathrm{~K}$, the behavior inside the thermal hysteresis loops, expressed by the FORC diagram, suffers strong modifications even in the presence of small pressure gradients.
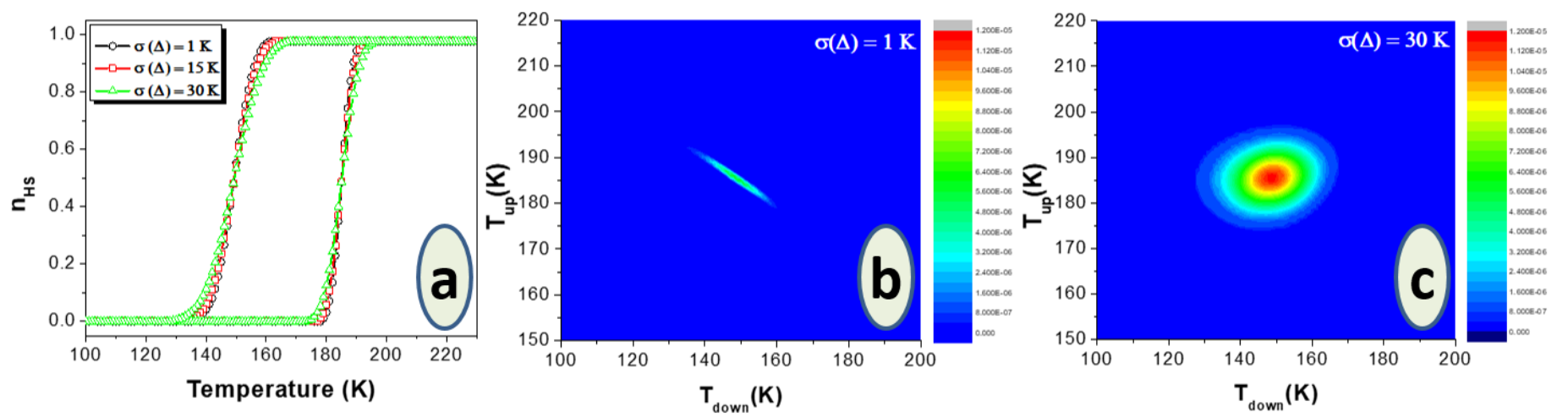

Figure 5. Comparative analysis of the pressure gradient effect on the major thermal hysteresis loop (a) and on the FORC diagram (b-c) simulated for various values of the pressure gradient. The following computational parameters have been used: $\Delta_{0}=1200 \mathrm{~K}, \Gamma_{0}=300 \mathrm{~K}$ and $\ln \mathrm{g}=7$.

\section{Conclusions}

In this work we analyzed the effect of a non-hydrostatic pressure on the spin transition properties of molecular spin crossover complexes in terms of major thermal hysteresis loop and FORC diagram methods. We have shown 
that for high pressure gradients, the thermal hysteresis loop becomes asymmetric. Remarkably, the FORC diagram method has been proved to be much more sensitive to local changes than the "bulk" parameters, which characterize the major hysteresis loop. This makes the FORC diagram method a suitable technique to characterize the hydrostaticity degree of a pressure cell. These results aim to shed light on a series of reported results on the pressure effect on spin crossover materials and on hysteretic properties of spin-crossover microparticles embedded in glass-forming matrices, analyzed at cryogenic temperatures, below the solidification of the medium surrounding the SCO particles.

\section{SUPPLEMENTARY MATERIAL}

See supplementary material for the superconducting transition of a bulk $\mathrm{Pb}$ wire recorded at ambient pressure and under a pressure of ca. 7.7 kbar, in water and Daphne oil and for Heat capacity profiles for Daphne 7373.

\section{Acknowledgments}

This work was partially founded by the European Commission through the SPINSWITCH project (H2020MSCA-RISE-2016, Grant Agreement No. 734322). The financial support of EXCALIBUR project Contract No. 18 PFE/16.10.2018 is also acknowledged.

Conflicts of Interest: The authors declare no conflict of interest.

\section{References}

[1] A. Bousseksou, G. Molnar, L. Salmon, W. Nicolazzi, Molecular spin crossover phenomenon: recent achievements and prospects, Chem. Soc. Rev., 40 (2011) 3313-3335.

[2] M.A. Halcrow, Spin-Crossover Materials. Properties and Applications., John Wiley \& Sons, Ltd., 2013.

[3] C.P. Slichter, H.G. Drickamer, Pressure-Induced Electronic Changes in Compounds of Iron, J. Chem. Phys., 56 (1972) 2142-2160.

[4] H.G. Drickamer, C.W. Frank, Electronic Transitions and the High Pressure Chemistry and Physics of Solids, Chapman and Hall, London, 1973.

[5] E. Konig, G. Ritter, J. Waigel, H.A. Goodwin, The Effect of Pressure on the Thermal hysteresis of the $1^{\text {st }}$ Order Spin Transition in Bis (1,10-Phenanthroline-2-Carbaldhyde Phenylhydrazone) Iron (II) Complexes, Journal of Chemical Physics, 83 (1985) 3055-3061. 
[6] V. Ksenofontov, G. Levchenko, H. Spiering, P. Gutlich, J.F. Letard, Y. Bouhedja, O. Kahn, Spin crossover behavior under pressure of $\mathrm{Fe}(\mathrm{PM}-\mathrm{L})_{2}(\mathrm{NCS})_{2}$ compounds with substituted 2'-pyridylmethylene 4-anilino ligands, Chemical Physics Letters, 294 (1998) 545-553.

[7] Y. Garcia, P.J. van Koningsbruggen, R. Lapouyade, L. Fournes, L. Rabardel, O. Kahn, V. Ksenofontov, G. Levchenko, P. Gutlich, Influences of temperature, pressure, and lattice solvents on the spin transition regime of the polymeric compound [ Fe(hyetrz) $\left.{ }_{3}\right] \mathrm{A}_{2} \cdot 3 \mathrm{H}_{2} \mathrm{O}$ (hyetrz = 4-(2 '-hydroxyethyl)-1,2,4-triazole and A- = 3nitrophenylsulfonate), Chem. Mater., 10 (1998) 2426-2433.

[8] T. Granier, B. Gallois, J. Gaultier, J.A. Real, J. Zarembowitch, High-pressure single-crystal x-ray diffraction study of two spin-crossover iron(II) complexes: $\mathrm{Fe}(\mathrm{Phen})_{2}(\mathrm{NCS})_{2}$ and $\mathrm{Fe}(\mathrm{Btz})_{2}(\mathrm{NCS})_{2}$, Inorganic Chemistry, 32 (1993) 5305-5312.

[9] P. Guionneau, C. Brigouleix, Y. Barrans, A.E. Goeta, J.F. Letard, J.A.K. Howard, J. Gaultier, D. Chasseau, High pressure and very low temperature effects on the crystal structures of some iron(II) complexes, Comptes Rendus De L Academie Des Sciences Serie Ii Fascicule C-Chimie, 4 (2001) 161-171.

[10] H.J. Shepherd, S. Bonnet, P. Guionneau, S. Bedoui, G. Garbarino, W. Nicolazzi, A. Bousseksou, G. Molnar, Pressure-induced two-step spin transition with structural symmetry breaking: X-ray diffraction, magnetic, and Raman studies, Physical Review B, 84 (2011) 144107.

[11] H.J. Shepherd, P. Rosa, L. Vendier, N. Casati, J.-F. Letard, A. Bousseksou, P. Guionneau, G. Molnar, High-pressure spin-crossover in a dinuclear Fe(II) complex, Phys. Chem. Chem. Phys., 14 (2012) 5265-5271.

[12] V. Legrand, F. Le Gac, P. Guionneau, J.F. Letard, Neutron powder diffraction studies of two spin transition Fe-II complexes under pressure, Journal of Applied Crystallography, 41 (2008) 637-640.

[13] A. Tissot, H.J. Shepherd, L. Toupet, E. Collet, J. Sainton, G. Molnar, P. Guionneau, M.L. Boillot, Temperature- and Pressure-Induced Switching of the Molecular Spin State of an Orthorhombic Iron(III) SpinCrossover Salt, Eur. J. Inorg. Chem., (2013) 1001-1008.

[14] C. Roux, J. Zarembowitch, J.P. Itie, A. Polian, M. Verdaguer, Pressure-induced spin-state crossovers in six-coordinate $\mathrm{Fe}(\mathrm{II}) \mathrm{L}_{n} \mathrm{~L}_{\mathrm{m}}(\mathrm{NCS})_{2}$ complexes with $\mathrm{L}=\mathrm{L}$ ' and $\mathrm{L}$ not equal L': A XANES investigation, Inorganic Chemistry, 35 (1996) 574-580.

[15] M.L. Boillot, J. Zarembowitch, J.P. Itie, A. Polian, E. Bourdet, J.G. Haasnoot, Pressure-induced spin-state crossovers at room temperature in iron(II) complexes: comparative analysis; a XANES investigation of some new transitions, New Journal of Chemistry, 26 (2002) 313-322.

[16] G. Molnar, T. Kitazawa, L. Dubrovinsky, J.J. McGarvey, A. Bousseksou, Pressure tuning Raman spectroscopy of the spin crossover coordination polymer $\mathrm{Fe}\left(\mathrm{C}_{5} \mathrm{H}_{5} \mathrm{~N}\right)_{2}\left[\mathrm{Ni}(\mathrm{CN})_{4}\right]$, Journal of Physics-Condensed Matter, 16 (2004) S1129-S1136. 
[17] G. Molnar, V. Niel, J.A. Real, L. Dubrovinsky, A. Bousseksou, J.J. McGarvey, Raman spectroscopic study of pressure effects on the spin-crossover coordination polymers Fe(pyrazine) $\left[\mathrm{M}(\mathrm{CN})_{4}\right] \cdot 2 \mathrm{H}_{2} \mathrm{O}(\mathrm{M}=\mathrm{Ni}, \mathrm{Pd}, \mathrm{Pt})$. First observation of a piezo-hysteresis loop at room temperature, Journal of Physical Chemistry B, 107 (2003) 3149-3155.

[18] J. Jeftic, U. Kindler, H. Spiering, A. Hauser, Helium gas pressure cell for pressures up to $1 \mathrm{kbar}(0.1 \mathrm{GPa})$ in conjunction with the cold head of a closed-cycle He refrigerator, Measurement Science \& Technology, 8 (1997) 479-483.

[19] E. Codjovi, N. Menendez, J. Jeftic, F. Varret, Pressure and temperature hysteresis in the spin-transition solid $\mathrm{Fe}(\mathrm{btr})_{2}(\mathrm{NCS})_{2} \cdot \mathrm{H}_{2} \mathrm{O}$, pure and diluted in Ni matrix, Comptes Rendus De L'Academie Des Sciences Serie II Fascicule C-Chimie, 4 (2001) 181-188.

[20] R. Tanasa, A. Stancu, J.F. Letard, E. Codjovi, J. Linares, F. Varret, Piezo- and thermo-switch investigation of the spin-crossover compound [Fe(PM-BiA $\left.)_{2}(\mathrm{NCS})_{2}\right]$, Chem. Phys. Lett., 443 (2007) 435-438.

[21] A. Rotaru, F. Varret, E. Codjovi, K. Boukheddaden, J. Linares, A. Stancu, P. Guionneau, J.F. Letard, Hydrostatic pressure investigation of the spin crossover compound [Fe(PM-BiA $\left.)_{2}(\mathrm{NCS})_{2}\right]$ polymorph I using reflectance detection, J. Appl. Phys., 106 (2009) 053515.

[22] C.-M. Jureschi, I. Rusu, E. Codjovi, J. Linares, Y. Garcia, A. Rotaru, Thermo- and piezochromic properties of $\left[\mathrm{Fe}(\right.$ hyptrz) $] \mathrm{A}_{2} \cdot \mathrm{H}_{2} \mathrm{O}$ spin crossover $1 \mathrm{D}$ coordination polymer: Towards spin crossover based temperature and pressure sensors, Physica B: Condensed Matter, 449 (2014) 47-51.

[23] A. Rotaru, J. Linares, F. Varret, E. Codjovi, A. Slimani, R. Tanasa, C. Enachescu, A. Stancu, J. Haasnoot, Pressure effect investigated with first-order reversal-curve method on the spin-transition compounds $\mathrm{Fe}_{\mathrm{x}} \mathrm{Zn}_{1-}$ x (btr $)_{2}(\mathrm{NCS})_{2} \cdot \mathrm{H}_{2} \mathrm{O}(\mathrm{x}=0.6,1)$, Phys. Rev. B, 83 (2011) 224107.

[24] G.G. Levchenko, G.V. Bukin, A.B. Gaspar, J.A. Real, The pressure-induced spin transition in the Fe(phen)2(NCS)2 model compound, Russian Journal of Physical Chemistry B, 83 (2009) 951-954.

[25] G. Levchenko, G. Bukin, H. Fylymonov, Q.J. Li, A.B. Gaspar, J.A. Real, Electrical Voltage Control of the Pressure-Induced Spin Transition at Room Temperature in the Microporous 3D Polymer Fe(pz) $\mathrm{Pt}(\mathrm{CN})_{4}$, Journal of Physical Chemistry C, 123 (2019) 5642-5646.

[26] V. Ksenofontov, G. Levchenko, H. Spiering, P. Gütlich, J.F. Létard, Y. Bouhedja, O. Kahn, Spin crossover behavior under pressure of $\mathrm{Fe}(\mathrm{PM}-\mathrm{L})_{2}(\mathrm{NCS})_{2}$ compounds with substituted 2'-pyridylmethylene 4-anilino ligands, Chemical Physics Letters, 294 (1998) 545-553.

[27] V. Ksenofontov, A.B. Gaspar, J.A. Real, P. Gutlich, Pressure-induced spin state conversion in antiferromagnetically coupled Fe(II) dinuclear complexes, Journal of Physical Chemistry B, 105 (2001) 1226612271. 
[28] P. Gutlich, A.B. Gaspar, V. Ksenofontov, Y. Garcia, Pressure effect studies in molecular magnetism, J. Phys. Condens. Matter, 16 (2004) S1087-S1108.

[29] P. Gutlich, V. Ksenofontov, A.B. Gaspar, Pressure effect studies on spin crossover systems, Coordination Chemistry Reviews, 249 (2005) 1811-1829.

[30] A. Galet, A.B. Gaspar, M.C. Munoz, G. Levchenko, J.A. Real, Pressure effect and crystal structure reinvestigations on the spin crossover system: $\left[\mathrm{Fe}(\mathrm{bt})_{2}(\mathrm{NCS})_{2}\right]\left(\mathrm{bt}=2,2^{\prime}\right.$-bithiazoline) polymorphs $\mathrm{A}$ and $\mathrm{B}$, Inorganic Chemistry, 45 (2006) 9670-9679.

[31] G. Agusti, A.L. Thompson, A.B. Gaspar, M.C. Munoz, A.E. Goeta, J.A. Rodriguez-Velamazan, M. Castro, R. Burriel, J.A. Real, Thermal, pressure and light induced spin transition in the two-dimensional coordination polymer $\left\{\mathrm{Fe}(\mathrm{pmd})_{2}\left[\mathrm{Cu}(\mathrm{CN})_{2}\right]_{2}\right\}$, Dalton Transactions, 2008 (2008) 642-649.

[32] A. Diaconu, S.-L. Lupu, I. Rusu, I.-M. Risca, L. Salmon, G. Molnár, A. Bousseksou, P. Demont, A. Rotaru, Piezoresistive Effect in the $\left[\mathrm{Fe}(\mathrm{Htrz})_{2}(\mathrm{trz})\right]\left(\mathrm{BF}_{4}\right)$ Spin Crossover Complex, J. Phys. Chem. Lett., 8 (2017) 3147-3151.

[33] K. Murata, K. Yokogawa, H. Yoshino, S. Klotz, P. Munsch, A. Irizawa, M. Nishiyama, K. Iizuka, T. Nanba, T. Okada, Y. Shiraga, S. Aoyama, Pressure transmitting medium Daphne 7474 solidifying at $3.7 \mathrm{GPa}$ at room temperature, Review of Scientific Instruments, 79 (2008) 085101.

[34] R. Tanasa, J. Laisney, A. Stancu, M.-L. Boillot, C. Enachescu, Hysteretic behavior of Fe(phen)2(NCS)2 spin-transition microparticles vs. the environment: A huge reversible component resolved by first order reversal curves, Applied Physics Letters, 104 (2014) 031909.

[35] R. Tanasa, C. Enachescu, J. Laisney, D. Morineau, A. Stancu, M.-L. Boillot, Unraveling the Environment Influence in Bistable Spin-Crossover Particles Using Magnetometric and Calorimetric First-Order Reverse Curves, The Journal of Physical Chemistry C, 123 (2019) 10120-10129.

[36] A. Bousseksou, J. Nasser, J. Linares, K. Boukheddaden, F. Varret, Ising-like model for the 2-step spin crossover, J. Phys. I, 2 (1992) 1381-1403.

[37] K. Boukheddaden, J. Linares, H. Spiering, F. Varret, One-dimensional Ising-like systems: an analytical investigation of the static and dynamic properties, applied to spin-crossover relaxation, Eur. Phys. J. B, 15 (2000) 317-326.

[38] D. Chiruta, C.-M. Jureschi, J. Linares, Y. Garcia, A. Rotaru, Lattice architecture effect on the cooperativity of spin transition coordination polymers, Journal of Applied Physics, 115 (2014) 053523.

[39] A.B. Gaspar, G. Molnár, A. Rotaru, H.J. Shepherd, Pressure effect investigations on spin-crossover coordination compounds, Compt. Rend. Chim., 21 (2018) 1095-1120. 
[40] A.P. Roberts, C.R. Pike, K.L. Verosub, First-order reversal curve diagrams: A new tool for characterizing the magnetic properties of natural samples, Journal of Geophysical Research-Solid Earth, 105 (2000) 2846128475.

[41] C.R. Pike, A.P. Roberts, K.L. Verosub, The effect of magnetic interactions on low temperature saturation remanence in fine magnetic particle systems, Journal of Applied Physics, 88 (2000) 967-974.

[42] C. Enachescu, R. Tanasa, A. Stancu, E. Codjovi, J. Linares, F. Varret, FORC method applied to the thermal hysteresis of spin transition solids: first approach of static and kinetic properties, Physica B-Condensed Matter, 343 (2004) 15-19.

[43] R. Tanasa, C. Enachescu, A. Stancu, J. Linares, E. Codjovi, F. Varret, Physical parameter distribution in spin transition systems derived from FORC data, Journal of Optoelectronics and Advanced Materials, 6 (2004) 551-556.

[44] C. Enachescu, R. Tanasa, A. Stancua, F. Varret, J. Linares, E. Codjovi, Kinetic hysteresis in spin crossover solids analyzed using FORC diagrams, Physica B-Condensed Matter, 372 (2006) 211-214.

[45] M.M. Dirtu, C. Neuhausen, A. Naik, A. Rotaru, L. Spinu, Y. Garcia, Insights into the Origin of Cooperative Effects in the Spin Transition of $\left[\mathrm{Fe}\left(\mathrm{NH}_{2} \operatorname{trz}\right)_{3}\right]\left(\mathrm{NO}_{3}\right)_{2}$ : the Role of Supramolecular Interactions Evidenced in the Crystal Structure of $\left[\mathrm{Cu}\left(\mathrm{NH}_{2} \mathrm{trz}\right)_{3}\right]\left(\mathrm{NO}_{3}\right)_{2} \cdot \mathrm{H}_{2} \mathrm{O}$, Inorganic Chemistry, 49 (2010) 5723-5736. [46] B. Weber, W. Bauer, T. Pfaffeneder, M.M. Dirtu, A.D. Naik, A. Rotaru, Y. Garcia, Influence of Hydrogen Bonding on the Hysteresis Width in Iron(II) Spin-Crossover Complexes, European Journal of Inorganic Chemistry, 2011 (2011) 3193-3206. 


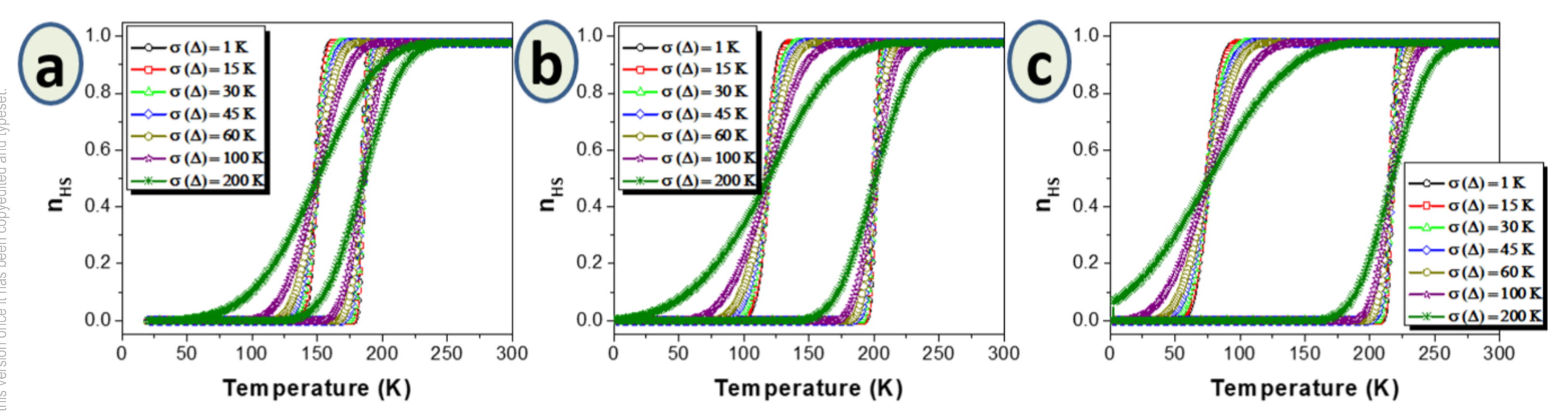



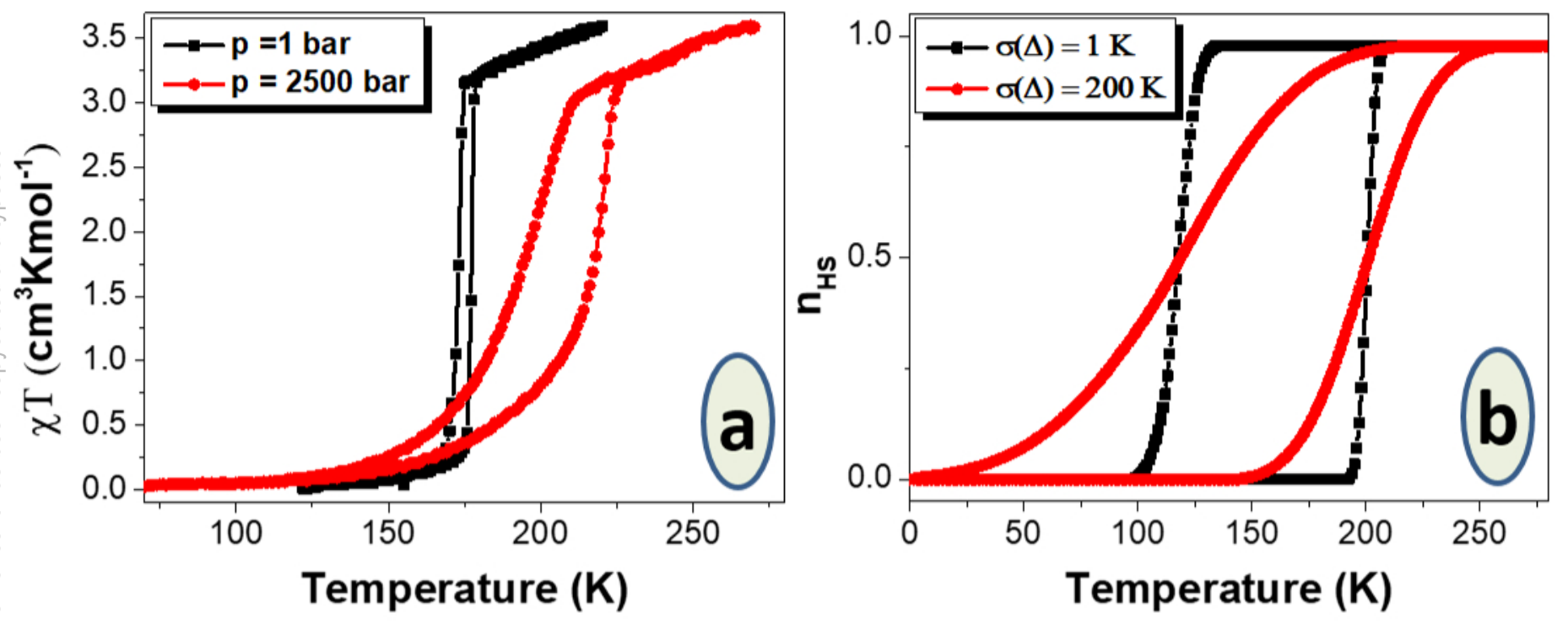

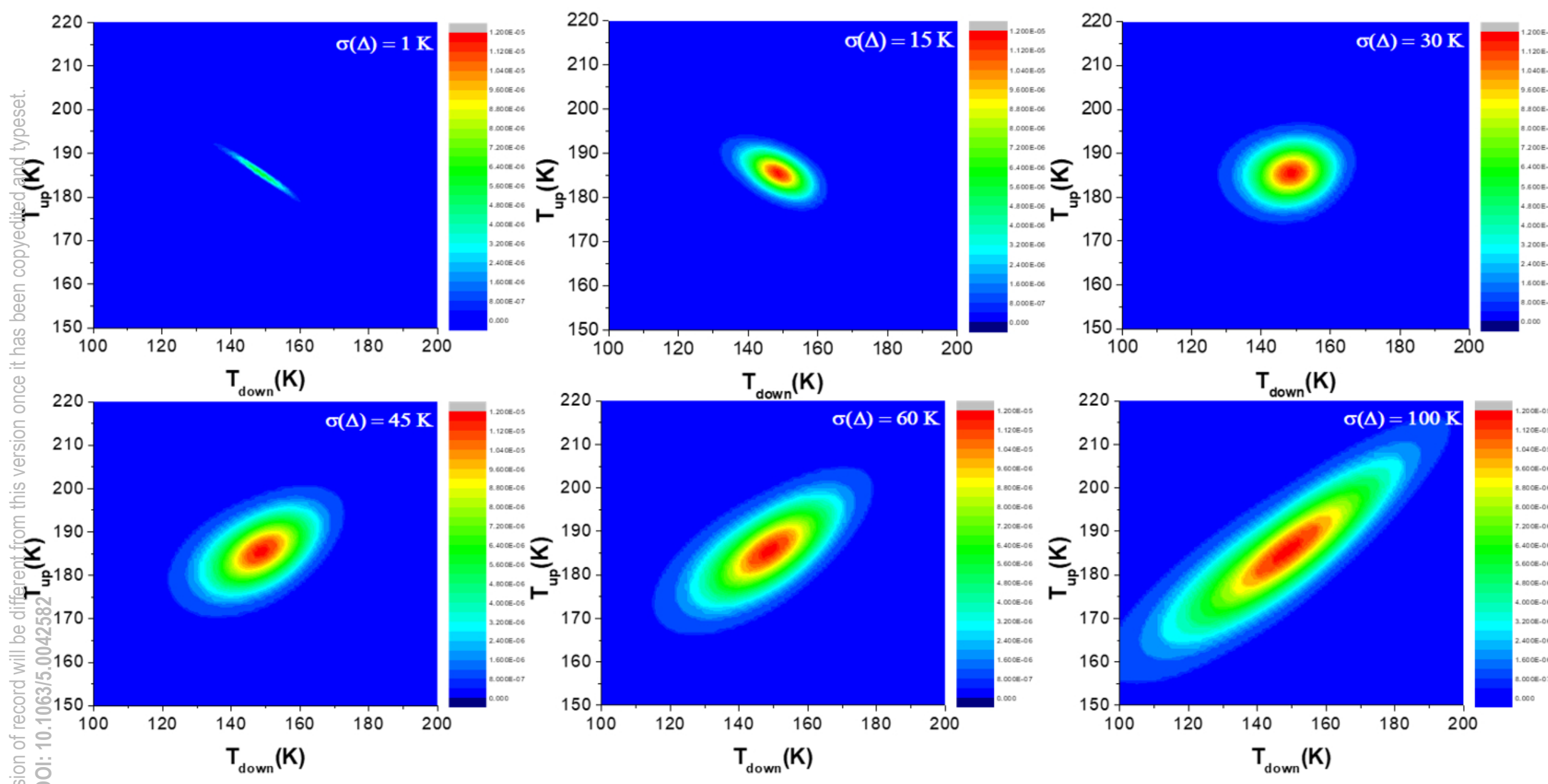

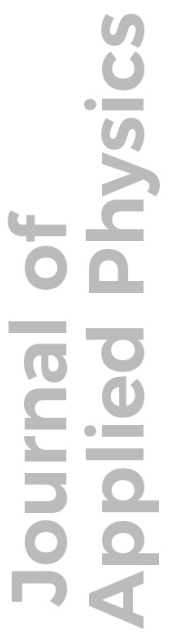

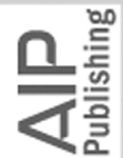




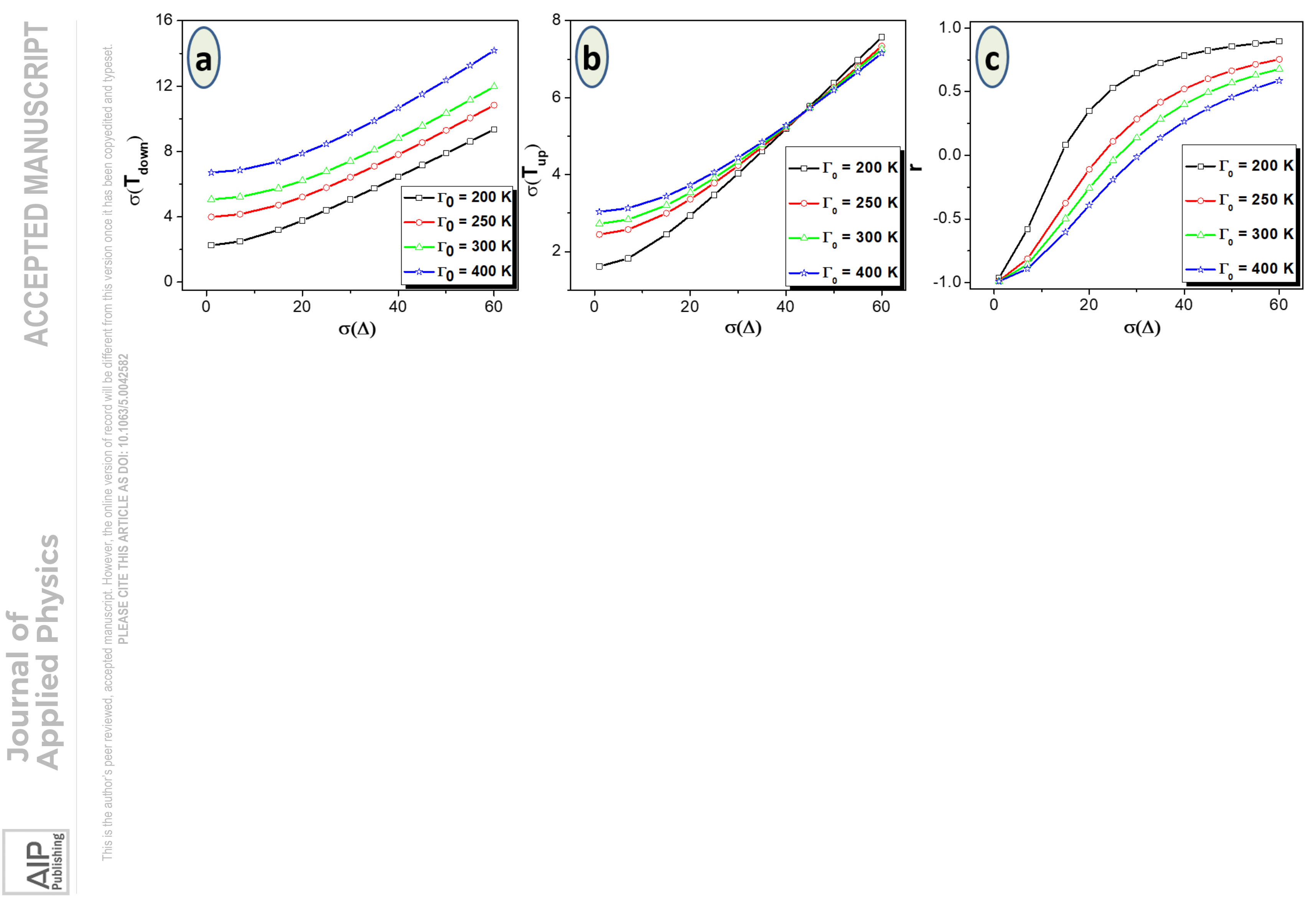




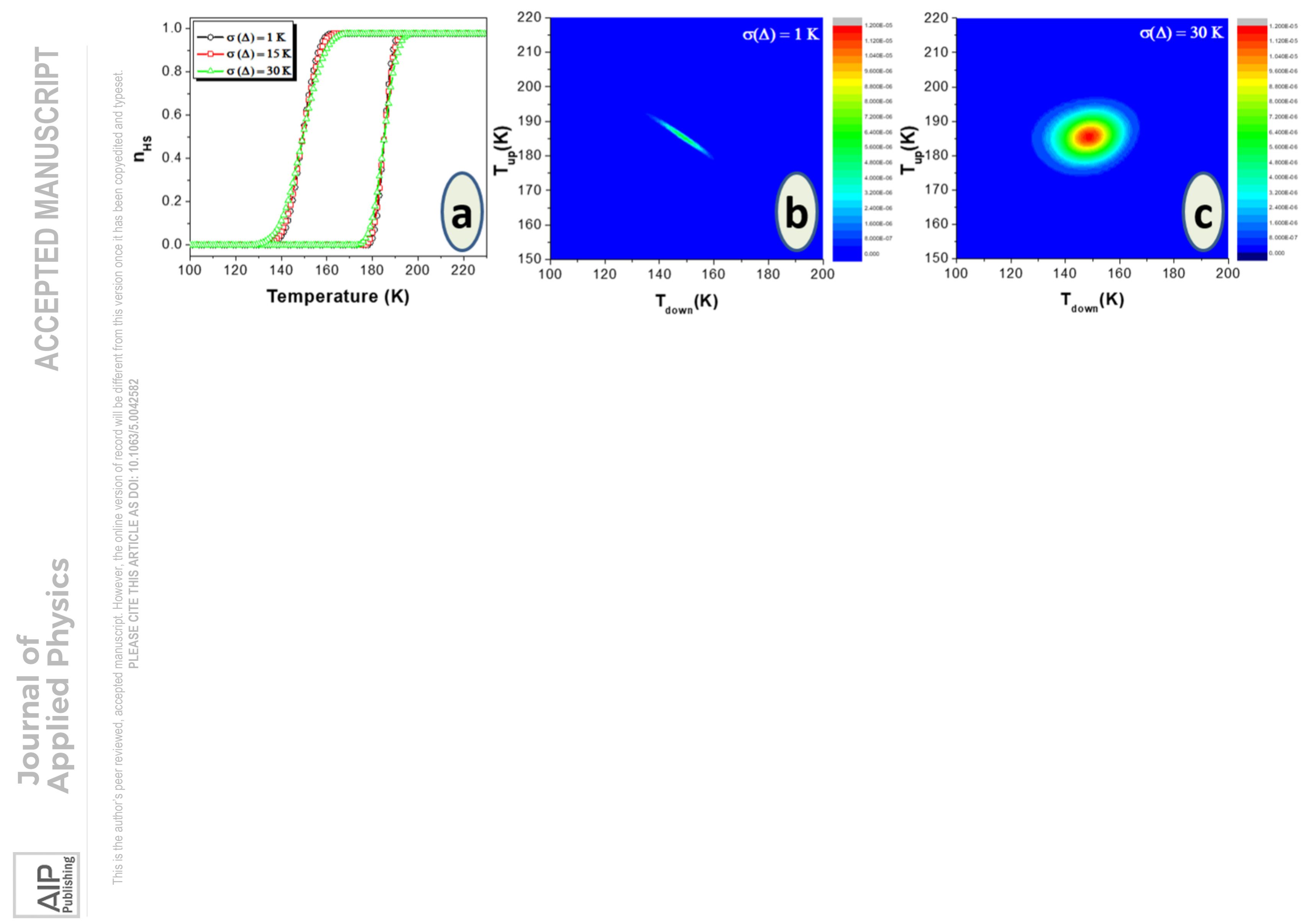

